\title{
Efeitos Estéricos e Eletrônicos de Substituintes Alquilas em Catalisadores Titanocênicos Solúveis na Polimerização Sindioespecífica de Estireno
}

\author{
Brasilio C. A. Pereira, Stanlei I. Klein e Marcos L. Dias
}

Resumo: Titanocenos são catalisadores solúveis conhecidos para a polimerisação estereoespecífica de olefinas pró-quirais como o estireno. Nesse trabalho descrevemos as relações entre as características do poliestireno e a estrutura do precursor do catalisador, de fato aqueles da família ( $\mathrm{RCp}$ ) ${ }_{2} \mathrm{TiCl}_{2}$ $(\mathrm{R}=\mathrm{H}$, etila, iso-propila, $n$-propila, sec-butila, $n$-butila, iso-amila e ciclohexila). Todos os catalisadores são ativos para a produção de poliestireno acima de zero graus centígrados. A sindiotaticidade dos polímeros são dependentes da cadeia lateral dos anéis aromáticos do titanoceno e da temperatura da polimerização. A relação entre os fatores estéricos e eletrônicos do precursor do catalisador e os produtos de polimerização são apresentados e discutidos.

Palavras-chave: Poliestireno, titanocenos, catálise Ziegler-Natta, catálise homogênea, efeitos estéricos e efeitos eletrônicos.

\section{Introdução}

Os catalisadores estudados neste trabalho estão baseados na substituição de um próton do anel ciclopentadieno, $\mathrm{Cp}$, por um grupo alquila. Da complexação desse ciclopentadienilo monosubstituído, RCp, a um sal de metal de transição, forma-se um complexo organometálico do tipo $(\mathrm{RCp})_{\mathrm{m}} \mathrm{MX}_{\mathrm{n}}$. Para o titânio (IV), a química desses compostos foi primeiro notificada por Sullivan e Little $^{[1]}$, e a primeira aplicação em catálise realizada por Hoecker e Saeki ${ }^{[2]}$. Na polimerização do estireno os titanocenos solúveis tem sido alvo de intensa investigação ${ }^{[3,4,5]}$. Porri e colaboradores, propuseram um mecanismo catalítico a partir de seus estu- dos sobre sistemas contendo compostos de titânio (III) e (IV) e metilalumoxano (MAO) como cocatalisador. Nessa proposta, o processo se inicia pela redução do catalisador pelo MAO. Em seguida, outra molécula de MAO provoca a substituição de um cloreto, remanescente, do complexo por um grupo metila gerando uma ligação metal carbono e seguindo-se a formação de um composto catiônico pela ação de uma terceira molécula de MAO.

Essa proposta mecanística será utilizada no presente trabalho para apoiar a argumentação sobre os resultados obtidos na polimerização do estireno por oito catalisadores de uma família de titanocenos do tipo $(\mathrm{RCp})_{2} \mathrm{TiCl}_{2}$ onde $\mathrm{R}=\mathrm{H}$, etila, $n$-propila, isopropila, $n$-butila, sec-butila, iso-amila e ciclo-hexila. 


$$
\begin{aligned}
& \mathrm{Cp}_{2} \mathrm{TiCl}_{2}+\mathrm{MAO} \longrightarrow \mathrm{Cp}_{2} \mathrm{TiCl} \\
& \mathrm{Cp}_{2} \mathrm{TiCl}+\mathrm{MAO} \longrightarrow \mathrm{Cp}_{2} \mathrm{TiCH}_{3} \\
& \mathrm{Cp}_{2} \mathrm{TiCH}_{3}+\mathrm{MAO} \longrightarrow\left[\mathrm{Cp}_{2} \mathrm{Ti}^{+}\left[\mathrm{MAOCH}_{3}\right]^{-}\right.
\end{aligned}
$$

Esquema 1. Mecanismo de ativação do complexo $\mathrm{Cp}_{2} \mathrm{TiCl}_{2}$ para polimerização de estireno.

\section{Experimental}

A síntese dos catalisadores foi baseada no método de Wilkinson e Birmingham?. A metodologia utilizada na polimerização do estireno, foi baseado nos trabalhos de Porri e colaboradores ${ }^{[5]}$. Em atmosfera inerte, na temperatura adequada $\left(0^{\circ} \mathrm{C}\right.$ ou $\left.50^{\circ} \mathrm{C}\right)$ e utilizando tolueno seco como solvente, foram adicionados, sob agitação, 8,2 $\mathrm{mL}$ de solução de metilalumoxano $0,5 \mathrm{M}$, a $5 \mathrm{~mL}$ de uma solução $3 \cdot 10^{-6}$ $\mathrm{M}$ de catalisador. Em seguida, o volume foi completando até $20 \mathrm{~mL}$ com tolueno e a mistura permaneceu sob agitação por 3 min para a formação do complexo ativado. A seguir, foram adicionados $5 \mathrm{~mL}$ do monômero à mistura que foi mantida sob agitação pelo período conveniente $\left(\right.$ a $0^{\circ} \mathrm{C}$ por um período de 18 horas, e a $50^{\circ} \mathrm{C}$ por 4 horas).

A reação foi interrompida pela adição cuidadosa de $200 \mathrm{~mL}$ de solução $3 \mathrm{M}$ de ácido clorídrico em etanol. O polímero obtido foi filtrado e lavado primeiramente com $10 \mathrm{~mL}$ da solução de $\mathrm{HCl}$ alcoólica e posteriormente com $20 \mathrm{~mL}$ de etanol puro. $\mathrm{O}$ sólido foi seco em estufa a $50^{\circ} \mathrm{C}$ por um período de 12 horas. O polímero bruto seco foi submetido a um processo de extração em soxhlet, com butanona-2 (MEC), por 6 horas.

Os dados de ressonância magnética foram obtidos a partir do Bruker AC 200-F. As curvas de calorimetria exploratória diferencial (DSC) foram adquiridas pelo Thermal Analyst 3100 da TA Instruments. Para os GPC, foi empregado o cromatógrafo Waters $150 \mathrm{CV}$ para trabalho a alta temperatura, equipado com detectores de índice de refração e viscosimétrico. Nesse cromatógrafo, foram utilizadas três colunas diferentes, mStyragel HT6, HT5 e HT4. A temperatura de trabalho foi de $135^{\circ} \mathrm{C}$, o solvente utilizado foi o triclorobenzeno numa vazão de $1 \mathrm{~mL} / \mathrm{min}$. A curva de calibração foi feita com padrões monodispersos de poliestireno.

\section{Resultados e Discussão}

$\mathrm{O}$ produto polimérico obtido apresentou-se como uma mistura de compostos que puderam ser separados em duas frações pela utilização de butanona-2 (MEC). A parte insolúvel apresentou ponto de fusão no intervalo $260^{\circ}$ a $278^{\circ} \mathrm{C}$ (Tabela 1), obtidos pela técnica de DSC, o que serviu para caracterizar essa fração como poliestireno sindiotático $^{[3]}$.

A análise por GPC desses polímeros insolúveis em butanona-2 revelou que as massas moleculares são relativamente altas. Para os polímeros obtidos nas reações efetuadas a $50^{\circ} \mathrm{C}$ essas massas variaram de 30000 a 43000 . O sistema catalítico empregado nesse trabalho favoreceu as reações de inserção versus reações de terminação de cadeia à baixa temperatura. Dessa forma os polímeros insolúveis produzidos a $0^{\circ} \mathrm{C}$ mostraram massas moleculares substancialmente mais elevadas, que variaram de 83000 a 129000 .

Essa fração do polímero bruto apresentou polidispersão (Mw/Mn), abaixo de 2 (Tabela 1). Esse valor é consistente com um processo polimérico onde o catalisador desenvolve um único tipo de sítio ativo. A microestrutura dessa fração insolúvel não foi completamente determinada.

Os espectros de r.m.n. de ${ }^{13} \mathrm{C}$ dos polímeros solúveis em MEC, obtidos em clorofórmio à temperatura ambiente, indicam uma mistura de polímeros atáticos, com sinais de maior intensidade na região de 145,2 ppm a 146,5 ppm (Figura 1), o que indica uma boa porcentagem de pentades regulares (sindiotáticas) ao longo das cadeias ${ }^{[6]}$.

$\mathrm{O}$ primeiro fato que chama a atenção quando da análise dos dados da Tabela 1 é a drástica redu-

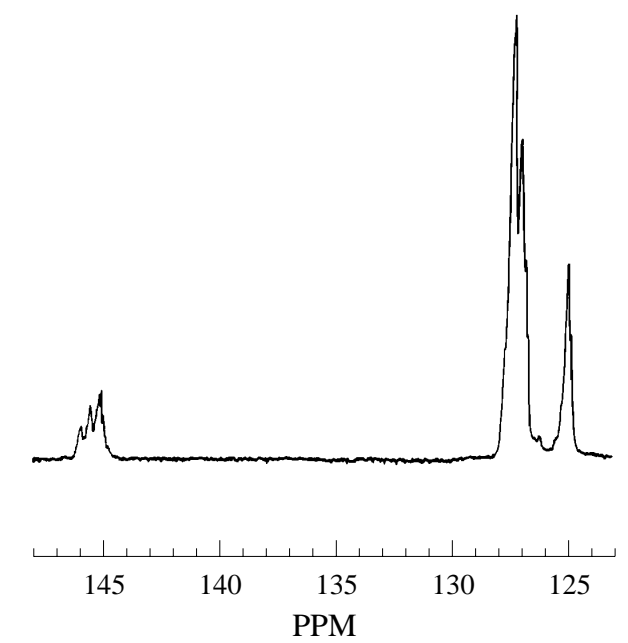

Figura 1. Espectro de r.m.n. de ${ }^{13} \mathrm{C}$ do polímero solúvel em butanona2 , obtido na reação cujo catalisador foi o $\left({ }^{\mathrm{s}} \mathrm{BuCp}\right)_{2} \mathrm{TiCl}_{2}$ na temperatura de trabalho de $50^{\circ} \mathrm{C}$. 
ção da massa bruta do polímero obtido, quando se passa do sistema $\mathrm{Cp}_{2} \mathrm{TiCl}_{2} / \mathrm{MAO}$ para o sistema $(\mathrm{RCp})_{2} \mathrm{TiCl}_{2} / \mathrm{MAO}$. Essa sensível diminuição do rendimento da polimerização deve certamente estar associada à introdução dos grupos $\mathrm{R}$ nos complexos; segundo o mecanismo proposto por Porri ${ }^{[5]}$, fica intuitivo deduzir que em $(\mathrm{RCp})_{2} \mathrm{TiCl}_{2}$ existe um aumento de densidade eletrônica sobre o metal, proporcionado pelo simples aumento dos efeitos indutivos dos anéis $\mathrm{Cp}$ sobre o metal, provocado pela presença dos grupos alquila. Portanto, quanto maior a indução eletrônica, mais difícil se tornaria a primeira etapa de ativação $\left(\mathrm{d}^{0} \rightarrow \mathrm{d}^{1}\right)$ do precursor, o que para a família $(\mathrm{RCp})_{2} \mathrm{TiCl}_{2}$ provocaria uma sensível diminuição no número de centros ativos ${ }^{[4]}$, com a conseqüente diminuição da massa bruta de polímero quando comparada a massa obtida com o parente $\mathrm{Cp}_{2} \mathrm{TiCl}_{2}$ em condições similares.

No presente trabalho, foi muito importante notar que os diferentes efeitos indutivos dos diferentes grupos $\mathrm{R}$, impõe aos complexos que compõem a família $(\mathrm{RCp})_{2} \mathrm{TiCl}_{2}$, atividades catalíticas sutilmente diferentes. Essa sutil porém perceptível diferença pode ser claramente relacionada ao tipo de indução causada por R. Em nosso caso, é possível subdividir a família de compostos $(\mathrm{RCp})_{2} \mathrm{TiCl}_{2} \mathrm{em}$ duas sub-famílias, uma delas possuindo um grupo $\mathrm{R}$ com um $\mathrm{CH}_{2} \alpha$ ao anel ( $\mathrm{R}=$ etila, $n$-propila, $n$ butila e iso-amila), que deveria ser a família contendo os metais com maior densidade eletrônica, devido aos efeitos indutivos, do que os metais da sub-família que possuiriam um $\mathrm{CH} \alpha$ ao anel $(\mathrm{R}=$ iso-propila, sec-butila e ciclo-hexila).

Assim sendo, era de se esperar que o grupo de complexos com $\mathrm{CH}_{2} \alpha$ ao anel $\left(\mathrm{RCH}_{2}-\right)$ produzisse uma quantidade menor de polímero quando comparado aos análogos do grupo de compostos com $\mathrm{R}$ com um $\mathrm{CH} \alpha$ ao anel $\left(\mathrm{R}_{2} \mathrm{CH}-\right)$, caso o efeito indutivo fosse o único efeito em operação. Surpreendentemente, os dados mostrados na Tabela 1 , obtidos das polimerizações efetuadas a $50^{\circ} \mathrm{C}$, indicam justamente uma situação inversa. Para os complexos com $\mathrm{RCH}_{2}-$, foram obtidas quantidades superiores de massa bruta de polímero em relação aos complexos com RCH-. Essa característica pode indicar que, para complexos com $\mathrm{RCH}_{2}$, a pequena desvantagem na catálise, esperada devido a efeitos indutivos, é superada por uma maior influência estérica.

Um efeito muito interessante da influência estérica do grupo substituinte $\mathrm{R}$ em compostos do

Tabela 1. Resultados da polimerização com $(\mathrm{RCp})_{2} \mathrm{TiCl}_{2}$ e análise dos polímeros.

\begin{tabular}{|c|c|c|c|c|c|c|c|c|}
\hline \multirow{2}{*}{ Composto } & \multirow{2}{*}{$\begin{array}{c}\mathbf{T}_{\mathbf{p}}^{*} \\
\left({ }^{\circ} \mathbf{C}\right)\end{array}$} & \multirow{2}{*}{$\begin{array}{l}\mathbf{M}_{\mathbf{p}}^{\#} \\
(\mathbf{m g})\end{array}$} & \multirow[b]{2}{*}{$\%$} & \multicolumn{2}{|c|}{ Fração insolúvel } & \multirow[b]{2}{*}{$\mathbf{T m}$} & \multicolumn{2}{|c|}{ Fração solúvel } \\
\hline & & & & Mw & Mw/Mn & & Mw & Mw/Mn \\
\hline \multirow{2}{*}{$\mathrm{Cp}_{2} \mathrm{TiCl}_{2}$} & 50 & 552 & 74 & 30600 & 1,34 & 260 & 9700 & 3,27 \\
\hline & 0 & 56 & 25 & 90600 & 1,42 & 264 & 12900 & 2,72 \\
\hline \multirow{2}{*}{$(\mathrm{EtCp})_{2} \mathrm{TiCl}_{2}$} & 50 & 120 & 51 & 34300 & 1,82 & 268 & 3300 & 1,75 \\
\hline & 0 & 48 & 56 & 129100 & 1,52 & 272 & 11800 & 1,80 \\
\hline \multirow[b]{2}{*}{$\left({ }^{\mathrm{n}} \mathrm{PrCp}\right)_{2} \mathrm{TiCl}_{2}$} & 50 & 138 & 62 & 40900 & 1,78 & 269 & 4800 & \multirow{2}{*}{1,81} \\
\hline & 0 & 29 & 31 & 128000 & 1,57 & 272 & 13600 & \\
\hline \multirow{2}{*}{$\left({ }^{(} \mathrm{PrCp}\right)_{2} \mathrm{TiCl}_{2}$} & 50 & 97 & 42 & 47600 & 1,75 & 269 & 5000 & 2,21 \\
\hline & 0 & 102 & 30 & 124900 & 1,46 & 275 & 12500 & 1,91 \\
\hline \multirow{2}{*}{$\left({ }^{\mathrm{n}} \mathrm{BuCp}\right)_{2} \mathrm{TiCl}_{2}$} & 50 & 239 & 54 & 36000 & 1,35 & 267 & 7600 & 2,70 \\
\hline & 0 & 119 & 33 & 98600 & 1,35 & 273 & 11200 & 2,34 \\
\hline \multirow{2}{*}{$\left({ }^{\mathrm{s}} \mathrm{BuCp}\right)_{2} \mathrm{TiCl}_{2}$} & 50 & 174 & 22 & 46400 & 1,33 & 266 & 8500 & $2, .26$ \\
\hline & 0 & 72 & 13 & 90900 & 1,38 & 272 & 10000 & 2,25 \\
\hline \multirow{2}{*}{$\left({ }^{\mathrm{i}} \mathrm{AmCp}\right)_{2} \mathrm{TiCl}_{2}$} & 50 & 167 & 67 & 33100 & 1,53 & 266 & 4000 & 2,10 \\
\hline & 0 & 23 & 45 & 85800 & 1,60 & 274 & 9700 & 2,11 \\
\hline \multirow{2}{*}{$(\mathrm{ChCp})_{2} \mathrm{TiCl}_{2}$} & 50 & 161 & 22 & 43000 & 1,29 & 266 & 3900 & 2,10 \\
\hline & 0 & 46 & 21 & 83300 & 1,35 & 278 & 9300 & 2,09 \\
\hline
\end{tabular}

O tempo de polimerização foi de $4 \mathrm{~h}$ a $50{ }^{\circ} \mathrm{C}$ e de $16 \mathrm{~h} \mathrm{a} 0{ }^{\circ} \mathrm{C}$. A [Ti] $=6.10^{-7} \mathrm{M}$ e a proporção Al/Ti foi de $2700 / 1$.

* $\mathrm{T}_{\mathrm{P}}=$ temperatura de polimerização. ${ }^{\#} \mathrm{M}_{\mathrm{P}}=$ massa bruta de polímero obtido na polimerização. 
tipo $(\mathrm{RCp})_{2} \mathrm{TiCl}_{2}$, que pode estar associado aos resultados de polimerização acima, foi investigado por Strelets e colaboradores ${ }^{[9]}$, no estudo da eletro-redução de vários compostos daquela família, particularmente com $\mathrm{M}=\mathrm{Ti}$ e $\mathrm{Zr}$, e $\mathrm{R}=$ metila, iso-propila, terc-butila, ciclo-hexila e trimetilsilano. Eles observaram que o aumento da densidade eletrônica sobre o metal em compostos do tipo $(\mathrm{RCp})_{2} \mathrm{MCl}_{2}$ induzida pela introdução do substituinte $\mathrm{R}$, provoca uma maior dificuldade na eletroredução levando os potenciais de redução do primeiro e segundo elétrons para valores mais negativos em relação ao $\mathrm{Cp}_{2} \mathrm{MCl}_{2}$ (Tabela 2).

De acordo com a Tabela 2, Strelets e colaboradores observaram uma inversão na tendência de aumento do potencial de redução (a entrada do primeiro elétron, que irá se alojar no orbital $1 \mathrm{a}_{1}$, Figura 2a) quando o substituinte possuía um efeito estérico muito grande, como em $\mathrm{R}=$ terc-butila e trimetilsilila. Para justificar essa tendência, aqueles autores levantaram hipóteses, todas elas do ponto de vista dos efeitos do impedimento estérico de $\mathrm{R}$ sobre as características eletrônicas do complexo.

Dentre as hipóteses de Strelets e colaboradores ${ }^{[9]}$, uma delas está baseada nas localizações espaciais dos grupos $\mathrm{R}$ em $(\mathrm{RCp})_{2} \mathrm{TiCl}_{2}$, tal como determinadas por espectroscopia de raios-X. As estruturas cristalinas sugeridas para os compostos $(\mathrm{RCp})_{2} \mathrm{TiCl}_{2}$ estudados por Strelets com $\mathrm{R}=$ metila, etila, iso-propila e ciclo-hexila são mostradas na figura $2 \mathrm{~b}$ e para os mais volumosos $\mathrm{R}=$ terc-butila e trimetilsilano são mostradas na figura $2 \mathrm{c}$.

Observando a Figura 2, fica claro que quanto maior o volume do grupo $\mathrm{R}$ maior será a possibilidade de uma interação de ordem coulômbica entre o grupo alquila (positivado por conseqüência da doação de densidade eletrônica para o anel) e o orbital

Tabela 2. Potenciais de eletroredução* de $(\mathrm{RCp})_{2} \mathrm{TiCl}_{2}$ obtidos em $\mathrm{THF}^{[9]}$.

\begin{tabular}{|c|c|c|}
\hline Composto & $\begin{array}{c}\text { Potencial de redução } \\
\text { do } 1^{\circ} \text { elétron } \\
\left(\mathrm{E}_{1}^{0}\right) \mathrm{em} \mathrm{mV}\end{array}$ & $\begin{array}{c}\text { Potencial de redução } \\
\text { do } 2^{\circ} \text { elétron } \\
\left(E_{2}\right) \text { em } \mathrm{mV}\end{array}$ \\
\hline $\mathrm{Cp}_{2} \mathrm{TiCl}_{2}$ & $-0,86$ & $-2,10$ \\
\hline$(\mathrm{MeCp})_{2} \mathrm{TiCl}_{2}$ & $-0,93$ & $-2,12$ \\
\hline$(\mathrm{ChCp})_{2} \mathrm{TiCl}_{2}$ & $-0,95$ & $-2,13$ \\
\hline$\left({ }^{i} \mathrm{PrCp}\right)_{2} \mathrm{TiCl}_{2}$ & $-0,96$ & $-2,14$ \\
\hline$(\mathrm{BuCp})_{2} \mathrm{TiCl}_{2}$ & $-0,90$ & $-2,08$ \\
\hline$\left(\mathrm{Me}_{3} \mathrm{SiCp}\right)_{2} \mathrm{TiCl}_{2}$ & $-0,87$ & $-2,07$ \\
\hline
\end{tabular}

*Em referência ao Calomelanos.

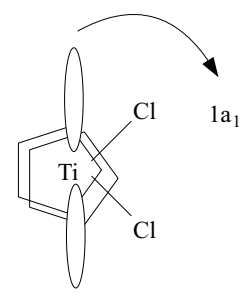

a

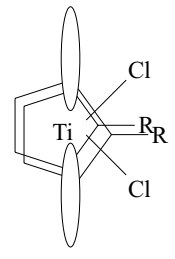

b

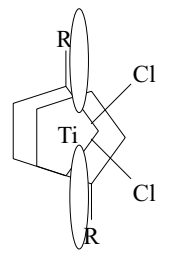

c
Figura 2. Estrutura cristalina dos compostos $(\mathrm{RCp})_{2} \mathrm{TiCl}_{2}$, a- para $\mathrm{R}$ $=$ metila, etila, iso-propila e ciclo-hexila (pouco volumosos); $\mathrm{b}$ para $\mathrm{R}=$ terc-butila e trimetilsilano (muito volumosos).

$1 \mathrm{a}_{1}$, permitindo que esse tipo de complexo (com $\mathrm{R}$ volumoso) sofra uma eletroredução para Ti(III) facilitada pela razoável interação $\mathrm{R}^{\delta+} \leftarrow 1 \mathrm{a}_{1}$ que seria desenvolvida. Assim sendo, a localização privilegiada do grupo alquila volumoso poderia ter um efeito coulômbico que seria mais importante, no tocante à processos de redução, que o seu próprio efeito indutivo. Isso é aparentemente confirmado quando se observa o potencial de redução do $2^{\circ}$ elétron, (Tabela 2) os compostos com os grupos mais volumosos são mais susceptíveis a receber um segundo elétron, tendo valores para essa segunda redução até menores que a do parente $\mathrm{Cp}_{2} \mathrm{TiCl}_{2}$.

Retomando o mecanismo catalítico ${ }^{[5]}$, temos que na primeira etapa o processo de redução química da espécie contendo Ti(IV) para Ti(III), se agora considerarmos às possíveis interações coulômbicas ${ }^{[8]}$ entre o fragmento alquila e o orbital $1 \mathrm{a}_{1}$, fica claro, pelos resultados obtidos (Tabela 1), que a geometria dos complexos contendo substituintes $\mathrm{RCH}_{2}$ - deva estar permitindo uma melhor interação entre $\mathrm{R}^{\delta+} \rightarrow 1 \mathrm{a}_{1}$, facilitando o mecanismo de ativação $\left(\mathrm{d}^{1} \rightarrow \mathrm{d}^{0}\right)$, proporcionando um número maior de centros ativos e consequentemente uma massa bruta polimérica maior.

Quando observamos a porcentagem de polímero insolúvel obtido em cada um dos casos, ainda à temperatura de $50^{\circ} \mathrm{C}$, a divisão da família sugerida acima fica muito clara. Comparativamente, obtivemos uma porcentagem maior de polímero insolúvel quando utilizamos $\mathrm{Cp}_{2} \mathrm{TiCl}_{2}$ ao invés da família $(\mathrm{RCp})_{2} \mathrm{TiCl}_{2}$. Aparentemente, aqui também o aumento da densidade eletrônica sobre o metal provoca uma diminuição na porcentagem de polímero insolúvel, Mantendo-se a divisão dos compostos em duas sub-famílias, os dados da Tabela 1 mostram que os substituídos com $\mathrm{RCH}_{2}$ produziram uma porcentagem de polímero insolúvel superior (51 a 67\%) àquela encontrada para 
os catalisadores com RCH- (22 a 42\%). Caso esse fato também seja relacionado à maior facilidade da primeira sub-família em ter o centro ativo estabilizado por atrações coulômbicas, o caráter estérico de $\mathrm{R}$ deve ser o que tem papel determinante nessa última comparação. O polímero insolúvel é o de cadeia mais estereorregular que, no presente caso possui características sindiotáticas (sPS).

A análise dos pesos moleculares dos polímeros insolúveis também mostrou uma característica interessante. Aparentemente, a família $(\mathrm{RCp})_{2} \mathrm{TiCl}_{2}$ proporciona em qualquer temperatura um significativo aumento do peso molecular do polímero em relação aos produtos das reações do protótipo $\mathrm{Cp}_{2} \mathrm{TiCl}_{2}$. Esse fenômeno pode estar relacionado também a efeitos relacionados aos grupos R. Ainda que eles atuem primariamente na diminuição do número de sítios ativos (efeito indutivo), o seu efeito estérico deve prejudicar de alguma forma as reações de terminação de cadeia, permitindo que um número maior de monômeros sejam introduzidos, o que resultaria em um polímero de maior massa molecular.

Por exemplo, nas polimerizações utilizando catalisadores de sítio único solúveis como os metalocenos, o principal processo de terminação é a "eliminação de hidrogênio $\beta$ ". A $\beta$-eliminação, só pode ocorrer se a cadeia for capaz de se aproximar do metal e interagir com ele através de uma interação $b$ agóstica. A presença do grupo $R$ parece minimizar essa interação impedindo de certa forma a ocorrência de reações de terminação através de $\beta$-eliminação.

Ainda no quesito de massa molecular dos polímeros formados, é aparente a subdivisão dos compostos substituídos em duas famílias distintas: os resultados de peso molecular dos polímeros insolúveis (sPS) a $50^{\circ} \mathrm{C}$, obtidos pela sub-família $\mathrm{RCH}_{2}$ - (33000 a 41000) e pela sub-família RR'CH(43000 a 48000), podem indicar que as possíveis interações coulômbicas que são facilitadas na primeira sub-família forçam também uma maior rigidez ao conjunto que suporta o centro ativo, o que permitiria intensificação das reações de terminação, por exemplo, impedindo a formação da cadeia polimérica no sentido de proporcionar à aproximação de hidrogênios-b que facilitariam os processos de eliminação de cadeia ( $\beta$-eliminação).

Os resultados de polimerizações efetuadas a $0^{\circ} \mathrm{C}$ mostram claramente uma menor formação de polímeros, quando comparadas às reações efetuadas a $50^{\circ} \mathrm{C}$, como esperado, uma vez que todos os processos de ativação do pré-catalisador tornam-se energeticamente proibitivos àquela temperatura, além de se tornarem mais lentas às próprias reações de iniciação, propagação e terminação de cadeias, típicas de tais sistemas. Mesmo assim, é de uma forma surpreendente encontrar, nos dados da Tabela 1 , os resultados de massa bruta de polímeros formados durante a ação catalítica dos compostos das sub-famílias dos complexos alquil-substituídos. Aquela sub-família contendo grupos $\mathrm{RCH}_{2}$ - produz massa polimérica bruta menor $(23 \mathrm{a} 48 \mathrm{mg}$, exceto $\mathrm{R}=n$-butila $119 \mathrm{mg}$ ), em contraste com a atividade daquela sub-família a $50{ }^{\circ} \mathrm{C}$, onde era ela a produtora de maior massa bruta de polímeros formados. É possível especular-se que o abaixamento da temperatura tem um efeito, sobre essa sub-família de compostos, de obrigar deslocamento do substituinte $\mathrm{R}$, de maior volume, para dentro da região espacial que envolve a mediatriz do ângulo Cl-Ti-Cl, em uma imitação dos efeitos observados no estado sólido para essa classe de organometálicos. Ora, essa situação pode remover os grupos $\mathrm{R}$ substituintes daquela posição média ótima de maximização dos efeitos coulômbicos, responsáveis pela estabilização dos intermediários da redução Ti(IV) $\rightarrow$ Ti(III). Esse efeito agora minimiza a formação de centros cataliticamente ativos, resultando em uma massa polimérica bruta menor. Uma vez que o efeito estérico/coulômbico parece ter sua importância minimizada pelo "efeito do estado sólido", é de se esperar que o efeito indutivo do substituinte R venha a se sobrepor, e que seus resultados, em relação à atuação do catalisador, seja espelhada nas propriedades do polímero formado durante a catálise. É necessário dizer que quaisquer comparações desse âmbito, dentro da família de compostos estudada no presente trabalho, pode se tornar contraditória; entretanto, podemos comparar compostos quase semelhantes, como por exemplo os dados oriundos da catálise promovida pelo $\left({ }^{\mathrm{n} P r C p}\right)_{2} \mathrm{TiCl}_{2}(\mathrm{Mw}=128000)$ e o parente $\mathrm{RCH}$ $\left({ }^{\mathrm{PrCp}}\right)_{2} \mathrm{TiCl}_{2}(\mathrm{Mw}=124900)$. Esses dados parecem indicar que, uma vez que a pequena quantidade de centros reduzidos seja efetivamente formada à temperatura de $0^{\circ} \mathrm{C}$, a densidade eletrônica sobre o metal seria tão incrementada, que grupos substituintes bom indutores teriam, novamente, maior capacidade de tomar parte na transferência coulômbica $\mathrm{R}^{\delta+} \leftarrow 1 \mathrm{a}_{1}$, minimizando o "efeito do estado sólido", e permi- 
tindo aos catalisadores se comportarem como o observado a $50^{\circ} \mathrm{C}$. É claro que, como poucos centros foram, naquelas condições, inicialmente formados, o rendimento em massa polimérica bruta continuaria sendo muito baixa. Em geral, os dados da Tabela 1 concordam com o argumento posto acima, quando comparadas as atividades de catalisadores com substituintes $\mathrm{R}$ com o mesmo número de átomos de carbono. É de se esperar também que, naquelas baixas temperaturas, as velocidades de reações colaterais de terminação de cadeia também sejam minimizadas, o que favoreceria a formação de cadeias poliméricas de mais alto peso molecular, independentemente do sistema sendo observado.

Outra tendência interessante a se ressaltar está na diminuição sistemática do peso molecular do polímero insolúvel no decorrer de nossa série a $0^{\circ} \mathrm{C}$, (Tabela 1). A hipótese que justifica tal comportamento é a atuação sistemática do "efeito do estado sólido" ao longo da série $\mathrm{R}=$ etila à ciclohexila. Assim, independentemente de outros efeitos de natureza eletrônica, os substituintes $\mathrm{R}$ teriam a tendência de cada vez mais se localizarem na região do espaço que compreende as ligações $\mathrm{TiCl}_{2}$, provocando um efeito estérico comprometedor da atividade catalítica geral dos complexos, à medida que aumenta o tamanho de $\mathrm{R}$.

\section{Conclusão}

A análise dos poliestirenos sindiotáticos obtidos por esses catalisadores à temperaturas de $0 \mathrm{e}$ $50^{\circ} \mathrm{C}$ indica ainda o efeito fundamental de um fator estérico que realça os efeitos eletrônicos através de um efeito coulômbico, que opera sobre os substituintes e os orbitais baseados no metal. Esse efeito coulômbico nunca havia sido relacionado à atividade catalítica de um metaloceno de titânio. Em todos os casos estudados, os polímeros formados pelos compostos substituídos apresentam maior peso molecular, maior polidispersão e , em princípio, maior estereoregularidade do que aquele formado pelo protótipo $\mathrm{Cp}_{2} \mathrm{TiCl}_{2}$.

$\mathrm{O}$ presente trabalho sugere que é possível "afinar" o catalisador para que ele reproduza o tipo de característica da cadeia polimérica para a produção de polímeros com qualidades específicas.

\section{Referências Bibliográficas}

1. Sullivan, N. F.; Little, W. F.; - J. Organomet. Chem. 8(2), 277. (196)

2 Hoecker, H.; Saeki, K.; - Makromol. Chem. 148, 107. (1971)

3 Dias, M. L.; Giarrusso, A.; Porri, L.; - Macromol. 26, 6664. (1993)

4 Grassi, A.; Pellecchia, C.; Oliva, L.; Laschi, F.; - Macromol. Chem. Phys. 196, 1093. (1995)

5 Ricci, G.; Bosisio, C.; Porri, L.; - Macromol.Rapid. Commun. 17, 781. (1996)

6 Wilkinson, G.; Pauson, P. L.; Birmingham, J. M.; Cotton, F. A.; - J. Am. Chem. Soc. 75, 1011. (1953)

7 Wilkinson, G.; Birmingham, J. M; - J. Am. Chem. Soc. 76, 4281. (1954)

8 Garbassi, F.; Gila, L.; Proto, A.; - Polymer News 19, 367. (1994)

9 Soloveichik, G.L.; Gavrilov, A. B.; Strelets, V. V.; - Metalloorg. Khim. 2, 2, 431. (1989) 